\title{
Study on the Use of Hydride Fuel in High-Performance Light Water Reactor Concept
}

\author{
Haileyesus Tsige-Tamirat and Luca Ammirabile \\ European Commission, JRC, Institute for Energy and Transport, Westerduinweg 3, 1755 LE Petten, Netherlands \\ Correspondence should be addressed to Luca Ammirabile; luca.ammirabile@ec.europa.eu
}

Received 18 November 2014; Revised 15 January 2015; Accepted 15 January 2015

Academic Editor: Iztok Tiselj

Copyright (C) 2015 H. Tsige-Tamirat and L. Ammirabile. This is an open access article distributed under the Creative Commons Attribution License, which permits unrestricted use, distribution, and reproduction in any medium, provided the original work is properly cited.

\begin{abstract}
Hydride fuels have features which could make their use attractive in future advanced power reactors. The potential benefit of use of hydride fuel in HPLWR without introducing significant modification in the current core design concept of the high-performance light water reactor (HPLWR) has been evaluated. Neutronics and thermal hydraulic analyses were performed for a single assembly model of HPLWR with oxide and hydride fuels. The hydride assembly shows higher moderation with softer neutron spectrum and slightly more uniform axial power distribution. It achieves a cycle length of 18 months with sufficient excess reactivity. At Beginning of Cycle the fuel temperature coefficient of the hydride assembly is higher whereas the moderator and void coefficients are lower. The thermal hydraulic results show that the achievable fuel temperature in the hydride assembly is well below the design limits. The potential benefits of the use of hydride fuel in the current design of the HPLWR with the achieved improvements in the core neutronics characteristics are not sufficient to justify the replacement of the oxide fuel. Therefore for a final evaluation of the use of hydride fuels in HPLWR concepts additional studies which include modification of subassembly and core layout designs are required.
\end{abstract}

\section{Introduction}

Among the design challenges for high-performance light water reactor (HPLWR) concept is the large axial density gradient of the working fluid supercritical water in the core which could exceed more than a factor of seven at the upper part of the core [1]. Therefore, the upper part of the core shows significant undermoderation which in turn leads to a lower power density. Several assembly design concepts have been proposed to mitigate the undermoderation. Most of the concepts consider the use of either water rods or solid zirconium hydride rods replacing fuel rods in the assembly. The current HPLWR design employs a square water channel $(26 \mathrm{~mm} \times 26 \mathrm{~mm})$, in which high-density water flows downwards, in the centre of the assembly to improve moderation. All these solutions reduce the fuel to moderator ratio and increase the heterogeneity of the core which in turn affects the performance of the core.

A fuel that contains a significant amount of moderator in a homogeneous mixture could also be used to mitigate the undermoderation. One such fuel is the hydride fuel $\mathrm{U}-\mathrm{ZrH}$ which can have hydrogen atomic density as high as water in LWR operation conditions. General Atomics Technologies Inc. has developed for its TRIGA research reactor the hydride fuel $\mathrm{U}-\mathrm{ZrH}_{1.6}$ which has been successfully used for several years [2]. A recent US study [3,4] has investigated the feasibility of the use of hydride fuels instead of commonly used oxide fuels in light water reactors to improve performance and safety. The study found that hydride fuels could safely operate in LWRs and among others could be employed to reduce undermoderation in BWR.

The objective of this paper is to evaluate the potential benefit of use of hydride fuel in the current subassembly design of the HPLWR without introducing significant modification. The relatively high concentration of hydrogen in the hydride fuel brings a gain in moderation ratio which could reduce the amount of additional moderator needed in the HPLWR concept. Using current HPLWR design data, neutronics and thermal hydraulic analyses were performed for a single assembly model with oxide and hydride fuels. The 
TABLE 1: HPLWR assembly design data.

\begin{tabular}{lc}
\hline Fuel rod outer diameter $[\mathrm{mm}]$ & 8.0 \\
$p / d$ (pitch to diameter ratio) & 1.18 \\
Cladding material & $\mathrm{SS} 316$ \\
Cladding thickness $[\mathrm{mm}]$ & 0.5 \\
Active core height $[\mathrm{mm}]$ & 4200 \\
Pressure $[\mathrm{MPa}]$ & 25 \\
Inlet temperature $[\mathrm{K}]$ & 583 \\
Inlet mass flux $\left[\mathrm{kg} / \mathrm{m}^{2} \mathrm{~s}\right]$ & 1100 \\
\hline
\end{tabular}

neutronics analyses are based on three-dimensional Monte Carlo calculations using latest continuous-energy nuclear data. For the thermal hydraulic analyses, a subchannel code with modification for the supercritical condition has been employed.

The current analyses are limited to Beginning of Cycle (BOC) conditions for a fresh assembly. For a final evaluation of the use of hydride fuels in the current HPLWR concept additional analyses at End of Cycle (EOC) conditions are indispensable.

\section{Approach}

2.1. Reference HPLWR Assembly. The current HPLWR design uses $645 \mathrm{~mm}$ square $7 \times 7$ fuel assemblies with $268 \mathrm{~mm}$ square $3 \times 3$ moderator box in the centre [5]. The assemblies are gathered to $3 \times 3$ assembly cluster with an interassembly gap for moderator flow between them. The number of fuel rods per assembly amounts to 40 . The fuel rod has a diameter of $8.0 \mathrm{~mm}$ with $0.5 \mathrm{~mm}$ cladding thickness. For radial separation of the fuel rods, wire wraps of diameter of $1.34 \mathrm{~mm}$ are used. The pitch size is $9.4 \mathrm{~mm}$ resulting in a $p / d$ ratio of about 1.18. The active length of the assembly is $4.2 \mathrm{~m}$ and the total assembly box length is $4.851 \mathrm{~m}$. A fission gas plenum of about $200 \mathrm{~mm}$ is provided both at the top and at the bottom of the fuel pin. The cladding and assembly box material is stainless steel SS316. The current design considers as a fuel $\mathrm{UO}_{2}$ with 5 $6 \%$ U-235 enrichment. A fuel density of $10.4 \mathrm{~g} / \mathrm{cm}^{3}$ is assumed corresponding to $95 \%$ of the theoretical density.

The reactor is assumed to operate at thermal power of $2540 \mathrm{MW}_{\text {th }}$ with a thermal efficiency of about $44 \%$. The operating pressure is $25 \mathrm{MPa}$ and the core inlet and outlet temperatures are $310^{\circ} \mathrm{C}$ and about $500^{\circ} \mathrm{C}$, respectively. The feed water enters the reactor pressure vessel above the core and is guided into the upper and lower plenum. The coolant from the lower plenum flows through the core in three passes: up (evaporator region) and downward (superheater I region) followed by final upward flow (superheater II region). From the upper plenum, moderator water flows downwards through the moderator box located in the centre of the assembly and through the interassembly gaps to the lower plenum where it mixes with fresh feed water to flow upwards. The coolant heats up by about $200^{\circ} \mathrm{C}$ as it flows through the core. The axial density of the coolant varies by a factor 7 from $0.75 \mathrm{~g} / \mathrm{cm}^{3}$ to $0.1 \mathrm{~g} / \mathrm{cm}^{3}$. The main HPLWR assembly design data are summarised in Table 1.
The neutronics and thermal hydraulic analyses were performed for a single assembly of the evaporator region assuming the abovementioned design and operation conditions. The geometry of the assembly is retained when using hydride fuel.

2.2. Hydride Fuel. Zirconium hydride is used as moderator and shielding material in various nuclear applications. Uranium dispersed in zirconium hydride $\mathrm{U}-\mathrm{ZrH}$ has been developed as a fuel for the TRIGA research reactor by General Atomics since 1957 [2], at the beginning as highly enriched fuel with $8.5 \mathrm{wt} \%$ uranium and later as a low enriched uranium (LEU) fuel containing up to $45 \mathrm{wt} \%$ uranium with $\mathrm{U}-235$ enrichment up to $20 \mathrm{wt} \%$. The hydrogen to zirconium ratio is nominally 1.6 .

The LEU TRIGA type fuel U- $\mathrm{ZrH}_{1.6}$ was developed to achieve inherent safety through high prompt negative reactivity coefficient. It exhibits also high fission product retention and chemical and dimensional stability over large swings of temperature. Further, it has significantly higher thermal conductivity compared to the usual oxide fuel $\mathrm{UO}_{2}$. The TRIGA hydride fuel is compatible with water and stainless steel. The usual fuel rod has a diameter of $12.95 \mathrm{~mm}$ with $0.406 \mathrm{~mm}$ Incoloy 800 cladding and active length of $559 \mathrm{~mm}$.

The steady-state fuel temperature for normal operation is limited to $750^{\circ} \mathrm{C}$ [2] which could be a serious limitation for use in HPLWR. The recent US study [3] has established that the TRIGA type fuel U- $\mathrm{ZrH}_{1.6}$ can safely operate in LWR at comparable linear heat generation rate level $(\sim 50 \mathrm{~kW} / \mathrm{m})$ to that attainable with oxide fuel and higher discharge burnup $(\sim$ $80 \mathrm{MWd} / \mathrm{kg}$ ). In general terms hydride fuel application needs to be verified in the operating conditions of HPLWR.

The usual TRIGA type fuel $\mathrm{U}-\mathrm{ZrH}_{1.6}$ contains up to $2.8 \mathrm{wt} \%$ erbium as burnable poison to compensate for reactivity swings. The theoretical density of $\mathrm{U}-\mathrm{ZrH}_{1.6}$ with $45 \mathrm{wt} \%$ uranium $\left(19.05 \mathrm{~g} / \mathrm{cm}^{3}\right)$ and $55 \mathrm{wt} \% \mathrm{ZrH}_{1.6}\left(5.64 \mathrm{~g} / \mathrm{cm}^{3}\right)$ amounts to $8.29 \mathrm{~g} / \mathrm{cm}^{3}$. The atomic density of hydrogen in $\mathrm{U}$ $\mathrm{ZrH}_{1.6}$ is $4.8 \times 10^{-2}$ atoms/barn-cm which is equal to that of LWR coolant under normal operation condition. Due to the low uranium content of hydride fuel compared to oxide fuel, hydride fuels require higher enrichment, in LEU fuel up to 20 wt $\%$ U-235.

There are different options to incorporate hydride fuel in the current HPLWR design: partial or complete replacement of oxide fuel rods by hydride fuel rods or replacement of the moderator box by hydride fuel rods. After initial assessment of these options, the present work considers the complete replacement option retaining the geometry and other design conditions.

\section{Computational Method}

3.1. Neutronics Calculations. Neutron transport calculations were performed with the Monte Carlo transport code MCNP5 [6] using nuclear data from the Joint Evaluated Fission Fusion File (JEFF3.1). Burnup calculations were performed using the beta version of the Monte Carlo transport code MCNPX2.6 [7]. 


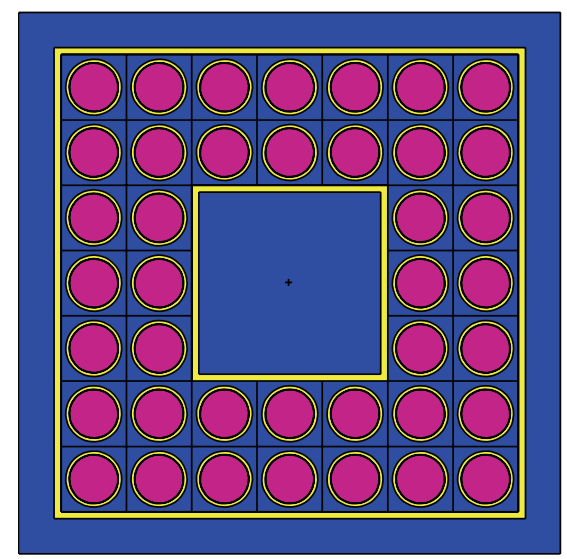

FIGURE 1: Horizontal cut of the HPLWR assembly MCNP model.

A three-dimensional MCNP geometry model of the HPLWR assembly has been set up based upon the data from the current HPLWR design as described in Section 2.1. A horizontal cut through the MCNP model at mid-plane is shown in Figure 1. The model includes beside the assembly the upper and lower plena which are modeled as homogeneous mixture of stainless steel SS316 and water which is subdivided in three axial zones of $20 \mathrm{~cm} / 50 \mathrm{~cm} / 100 \mathrm{~cm}$ with $80 \%$ SS316 and $20 \% \mathrm{H}_{2} \mathrm{O} / 50 \% \mathrm{SS} 316$ and $50 \% \mathrm{H}_{2} \mathrm{O} / 100 \% \mathrm{H}_{2} \mathrm{O}$ respectively. Neutron leakage in the axial direction is therefore properly accounted for. The model contains also upper and lower fission gas plenum as well as the assembly nozzle. In the axial direction the active core region is divided into 42 axial zones of $100 \mathrm{~mm}$ length to be able to represent the axial coolant density variation. The pitch size of the assembly is $9.4 \mathrm{~mm} \times 9.4 \mathrm{~mm}$. The pellet, gap, and cladding are included in the fuel rod model. The assembly box is surrounded by the interassembly moderator water. The reference enrichment for the oxide fuel assembly is $5 \mathrm{wt} \% \mathrm{U}-235$. For the assembly with hydride fuel, an enrichment of $11.11 \mathrm{wt} \%$ U-235 has been assumed to achieve a similar heavy metal (HM) weight in the zirconium hydride matrix.

MCNP calculations were performed assuming reflecting boundary conditions at the lateral sides of the assembly. The results are normalized to a power level of $1.81 \mathrm{MW}$ which is the average assembly power generation of the current design. For reactivity coefficient calculations, temperature dependent continuous-energy cross section data based on JEFF3.1 were used.

Sufficient numbers of particle histories and criticality cycles were simulated in the MCNP calculations to reduce statistical uncertainties. Thus, the relative statistical uncertainties of the results were mostly below $1 \%$. The calculations were performed in parallel using the MPI version of MCNP5 and MCNPX2.6 on a Linux cluster.

3.2. Thermal Hydraulic Calculations. The thermal hydraulic calculations were performed using the subchannel code COBRA-EN modified for supercritical water conditions. The COBRA-EN code [8] was originally developed in the eighties as an upgrade of the COBRA-3C/MIT code. The code was successively upgraded according to other computer codes, based on more refined and consistent features, like COBRAIV-I and, mainly, VIPRE-01, both evolved from the original COBRA-3C subchannel analysis code. COBRA-EN is used for transient and steady-state thermal hydraulic analyses of LWR assemblies and cores.

Fuller details of COBRA-EN upgrade to supercritical water conditions are given elsewhere [9]. We are limited in this section to recalling what the main code modifications are.

Above the critical pressure (22.064 MPa), water behaves essentially like a monophase fluid with a strong variation of its thermal-physical properties observed in the vicinity of the pseudocritical line. This large variation of thermalphysical properties has effects on the fluid-dynamic and heat transfer behavior. Therefore, the correct implementation of water property tables valid in the supercritical region is a prerequisite for an accurate analysis under prototypical HPLWR conditions. For this purpose, the IAPWS Industrial Formulation 1997 for the Thermodynamic Properties of Water and Steam [10] has been implemented in COBRA-EN for the water and steam thermodynamic property formulations.

The Bishop correlation [11] for the heat transfer coefficient was implemented in COBRA-EN to replace the DittusBoelter correlation [12] that was shown in several experimental studies [11,13] to be inadequate above the critical pressure of water. Indeed the Bishop correlation seems to be the most suitable for safety design studies, being able to predict supercritical water heat transfer well (none of the supercritical water heat transfer correlations appears to capture the deteriorated heat transfer phenomena observed with vertical tubes [14]) in the whole HPLWR operating range [15] and can also compute conservatively near the pseudocritical point [14].

The friction factor in turbulent flow at supercritical pressures was also modified on the basis of the review study of Cheng and Schulenberg [15]. The following equation was implemented for the friction factor:

$$
f=\left(1.82 \cdot \ln \left(\frac{\mathrm{Re}}{8}\right)\right)^{-2} \cdot\left(\frac{\rho_{w}}{\rho_{B}}\right)^{0.4},
$$

where $\rho_{w} / \rho_{B}$ is the ratio of density of water near the wall and its bulk value; $R e$ is the Reynolds number.

The fuel assembly is modelled in COBRA-EN in agreement with the MCNP model. The model consists of 40 fuel rods surrounded by 60 subchannels. The numbering for fuel rods and subchannels is indicated in Figure 2. The moderator channel which is hydraulically separated from the coolant subchannels, but thermally connected to them, is not simulated.

The COBRA-EN takes fully into account the subchannel mixing. Fuller details can be found in [8], but in essence the mixing is considered as follows.

Balance equations for the mixture mass and energy of the flowing coolant include the cross flow summation terms of the mass and enthalpy inflow or outflow in the lateral directions, while the balance equation for the mixture momentum vector is split into scalar equations for the axial 


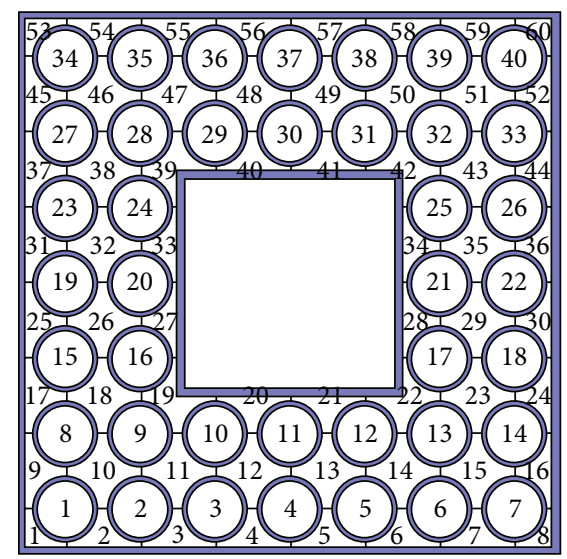

FIGURE 2: HPLWR subchannels model and fuel rods numbering.

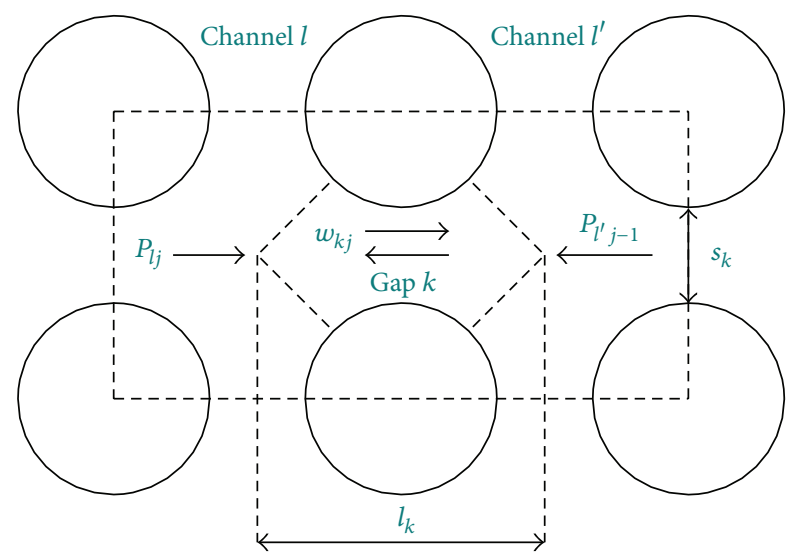

FIGURE 3: Control volume for lateral momentum balance finitedifference equation (top view).

momentum and for the lateral momentum in the directions dictated by the subchannel gap orientations (Figure 3).

The closure equations include the thermal conductance in lateral directions and the transverse turbulent mixing. The former equation is computed as

$$
C_{T}=\frac{G_{T} \bar{k}}{l_{k}}
$$

where $\bar{k}$ is arithmetic mean of the thermal conductivities for channels $i$ and $n$ as a function of the equation of state for supercritical water [10], $G_{T}$ is geometric factor for thermal conductance (squared array), and $l_{k}$ is gap lateral length.

The latter equation has the form of

$$
f_{T} \Delta X_{j} \sum_{k \in i} w_{k j}^{\prime}\left(U_{i j}^{\prime}-U_{n j}^{\prime}\right),
$$

where $f_{T}$ is transverse momentum factor, $\Delta X$ is axial length, $w^{\prime}$ is turbulent cross flow, and $U^{\prime}$ is the related effective momentum.

A transverse momentum factor of 0.02 is applied to account for the mixing behavior of the adjacent subchannels $[16,17]$.

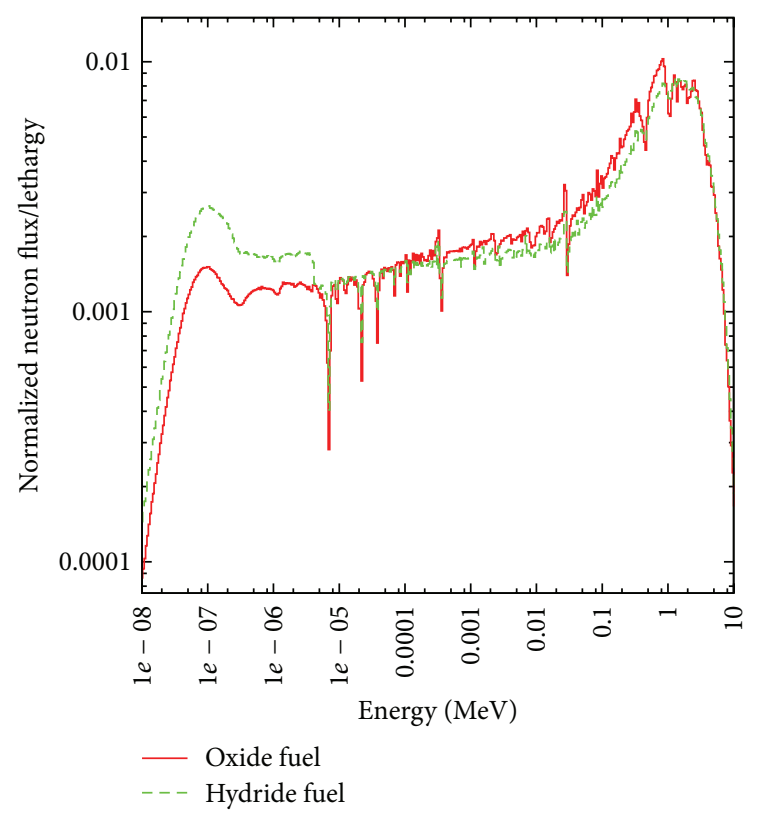

FIgURE 4: Normalized neutron energy spectra in the HPLWR assembly with oxide and hydride fuel.

The exit pressure of the fuel assembly is $25.0 \mathrm{MPa}$, and the water temperature at inlet is $583 \mathrm{~K}$.

\section{Results of Neutronic Analysis}

4.1. Neutron Multiplication and Fluxes. Neutronics analyses were performed for oxide and hydride fuel using the reference design data at BOC for fresh assemblies. With the reference enrichment of U-235 of $5 \mathrm{wt} \%$ in the oxide and $11.11 \mathrm{wt} \%$ in the hydride assembly, it is possible to achieve excess reactivity sufficient to sustain a cycle length of 18 months. The calculated BOC neutron multiplication factor $K_{\text {eff }}$ for oxide and hydride assemblies is 1.23 and 1.28 , respectively. The average neutron flux in the oxide assembly is $2.06 \times$ $10^{14} \mathrm{n} / \mathrm{cm}^{2} \mathrm{~s}$ and in the hydride assembly $1.67 \times 10^{14} \mathrm{n} / \mathrm{cm}^{2} \mathrm{~s}$.

The relatively high concentration of hydrogen in the hydride fuel increases the moderation in the hydride assembly significantly. Figure 4 shows the normalized neutron spectra calculated in ultrafine energy group structure in both oxide and hydride assembly options. The fluctuation in the depicted neutron spectra could be partially attributed to statistical errors which cannot be reduced everywhere in the ultrafine energy group structure. Due to the gain in moderation ratio, the neutron spectrum in the hydride assembly is considerably softer than in the oxide assembly. In the thermal and epithermal energy ranges, the group fluxes in the hydride core are up to a factor of two higher than in the oxide assembly.

Figure 5 compares the axial flux distribution in the oxide and hydride assemblies. The depicted flux distribution represents the radial average flux of an axial mesh in the active core region. In both assembly options the axial flux distribution achieves its maximum in the lower part of the assembly. This could be attributed to the higher hydrogen 


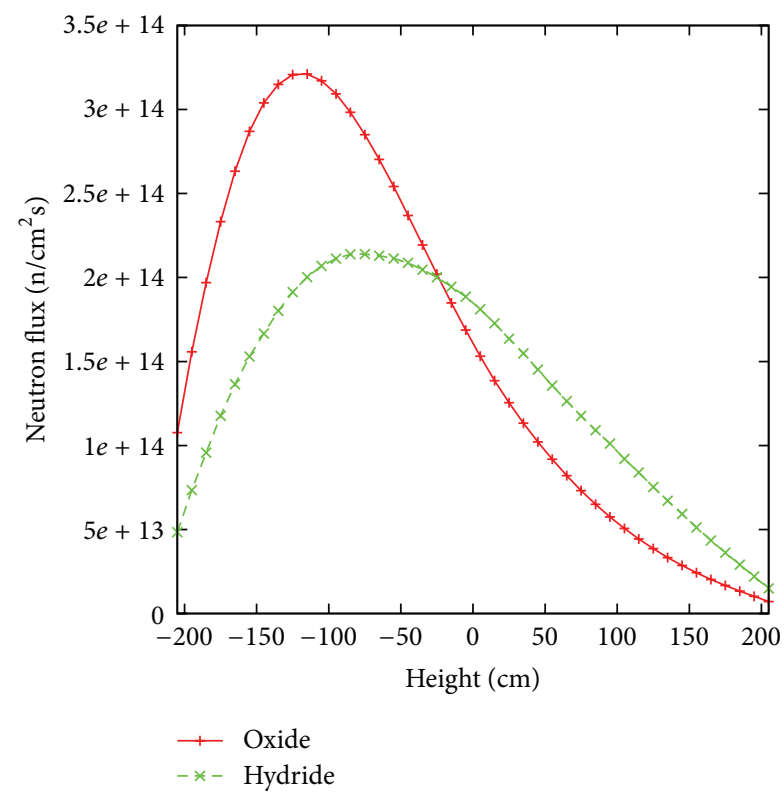

FIgUre 5: Axial neutron flux distribution in HPLWR with oxide and hydride assemblies.

density in this part. The axial power peaking factor in the oxide assembly is 1.56 and 1.23 in the hydride assembly.

The gain in moderation in the hydride assembly again leads to more uniform axial power distribution. However, in both assemblies the neutron flux drops by an order of a magnitude at the outermost position at the assembly outlet.

4.2. Reactivity Coefficients. To assess the neutronics safety behavior of the assembly options with oxide and hydride fuel, reactivity coefficients were calculated. The calculations are performed for fresh assemblies at BOC. Additional analyses at End of Cycle (EOC) conditions are indispensable since the depletion of U-235 and buildup of Pu isotopes may have a significant effect on certain reactivity coefficients such as the void coefficient. The definition of reactivity coefficients can be found in [18]. The moderator temperature reactivity feedback is the change in reactivity due to the change in the moderator temperature at constant density. The void reactivity feedback is the change in reactivity due to the change in density at constant temperature. The fuel temperature and moderator reactivity coefficients were calculated over a wide temperature range and the final results are obtained as averaged values. Similarly, the void coefficient was determined by averaging values calculated for different void fractions. The calculated reactivity coefficients are given in Table 2 .

The presence of the solid moderator $\mathrm{ZrH}_{1.6}$ in the fuel causes the hardening of the neutron spectrum with increasing temperature that combined with the Doppler effect leads to a more negative fuel temperature coefficient in the hydride assembly. The reason for this is the increased upscattering of thermal neutrons by gaining energy from excited hydrogen atoms in the zirconium hydride matrix [2].

The hydride assembly has lower moderator and void coefficients. Therefore, it is less sensitive to moderator
TABLE 2: Reactivity coefficients for oxide and hydride assemblies.

\begin{tabular}{lcc}
\hline & Oxide assembly & Hydride assembly \\
\hline $\begin{array}{l}\text { Fuel temperature } \\
\text { coefficient }[\mathrm{pcm} / \mathrm{K}]\end{array}$ & -2.43 & -3.90 \\
$\begin{array}{l}\text { Moderator coefficient } \\
{[\mathrm{pcm} / \mathrm{K}]}\end{array}$ & -15.1 & -4.48 \\
$\begin{array}{l}\text { Void coefficient } \\
\text { [pcm/25\% void] }\end{array}$ & -1590 & -625 \\
\hline
\end{tabular}

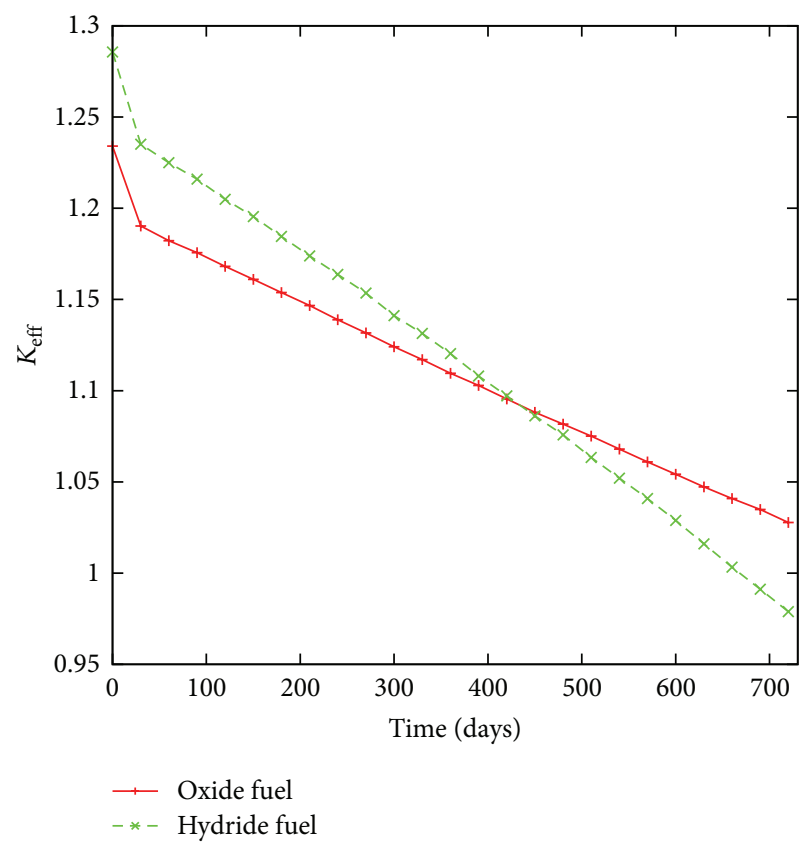

FIgURE 6: Time dependent $K_{\text {eff }}$ in HPLWR assembly with oxide and hydride fuel.

temperature changes and to voiding effects. This could also be partially attributed to the presence of the moderator in the fuel matrix.

4.3. Burnup. Reactivity swing due to fuel burnup has been analyzed in both oxide and hydride assemblies. The burnup calculations were performed using 63 energy group spectra for full power operation over a period of 24 months with output interval of 1 month.

The time dependent effective multiplication factor is depicted in Figure 6. Both assemblies could achieve a cycle length of 18 months. The reactivity loss proceeds faster in the hydride assembly due to the low heavy metal inventory. The EOC $K_{\text {eff }}$ in the oxide assembly is around 1.06 and that of the hydride assembly is 1.05 .

The discharge burnup of the hydride fuel after 18 months of full power irradiation is $41.9 \mathrm{GWd} / \mathrm{MTU}$. It is more than a factor of two higher than that of the oxide assembly which is about 16.7 GWd/MTU. The higher burnup is due to the higher enrichment of the hydride fuel compared with the oxide fuel. 
TABLE 3: Radial pin-power factor oxide assembly.

\begin{tabular}{lllllll}
\hline 1.161 & 1.012 & 0.981 & 0.980 & 0.981 & 1.012 & 1.161 \\
1.013 & 0.909 & 0.971 & 1.019 & 0.971 & 0.910 & 1.012 \\
0.981 & 0.971 & & & & 0.970 & 0.981 \\
0.980 & 1.019 & & & & 1.020 & 0.980 \\
0.981 & 0.971 & & & & 0.971 & 0.981 \\
1.013 & 0.910 & 0.971 & 1.019 & 0.971 & 0.910 & 1.013 \\
1.162 & 1.013 & 0.981 & 0.980 & 0.981 & 1.013 & 1.162 \\
\hline
\end{tabular}

TABLE 4: Radial pin-power factor hydride assembly.

\begin{tabular}{lllllll}
\hline 1.146 & 1.012 & 0.987 & 0.985 & 0.985 & 1.012 & 1.145 \\
1.011 & 0.917 & 0.972 & 1.015 & 0.972 & 0.916 & 1.011 \\
0.986 & 0.972 & & & & 0.972 & 0.985 \\
0.985 & 1.016 & & & & 1.015 & 0.984 \\
0.986 & 0.973 & & & & 0.971 & 0.984 \\
1.012 & 0.917 & 0.972 & 1.017 & 0.972 & 0.916 & 1.012 \\
1.144 & 1.012 & 0.985 & 0.985 & 0.986 & 1.011 & 1.144 \\
\hline
\end{tabular}

\section{Thermal Hydraulic Analysis}

5.1. Power Density Distribution. The pinwise radial distribution of the normalized power density in the two assemblies is given in Tables 3 and 4 . The fuel pins with maximal and minimal power are located in the corner and centre of fuel assembly, respectively. The pin-power factor in the oxide assembly varies from 0.91 to 1.162 , whereas in the hydride assembly it is in the range of 0.917 to 1.146 . The distribution in the hydride fuel is slightly more uniform.

The axial power profile is similar to the neutron flux profile which is described in Section 4.1.

5.2. Results of Thermal Hydraulic Analysis. Figures 7 and 8 show, respectively, the axial average coolant density and temperature over the active assembly height. For the steadystate analysis, the inlet water temperature is set at $583 \mathrm{~K}$. Since the geometry, the mass flow, and the total power are the same in both fuel assemblies, the average density and temperature remain the same at the core outlet. Following the axial power distribution, the axial density profile in the hydride assembly has a lower gradient. It should be pointed out that the coolant density can be affected by the moderator mass flow and the heat transfer condition for the moderator channels. This effect has not been taken into account during this analysis.

The hot channel factors (enthalpy rise in the subchannel over the average enthalpy rise) are shown in Tables 5 and 6. The maximum and minimum values are reached at the corner and the centre of fuel assembly, respectively. The power distribution in the hydride assembly has larger variation. The hot channel factor varies from 0.989 to 1.021 , whereas in the oxide assembly the pin-power factor is in the range of 0.995 to 1.014 .

The cladding temperature distribution of the hottest rods (oxide fuel rod 39; hydride fuel rod 7) in both assemblies is presented in Figure 9. Following the axial power distribution

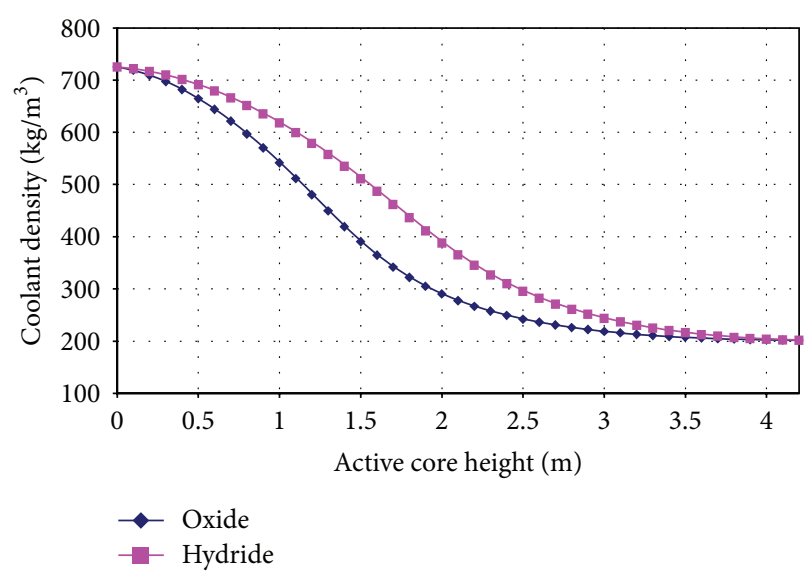

FIgURE 7: Average axial density profile in oxide and hydride assemblies.

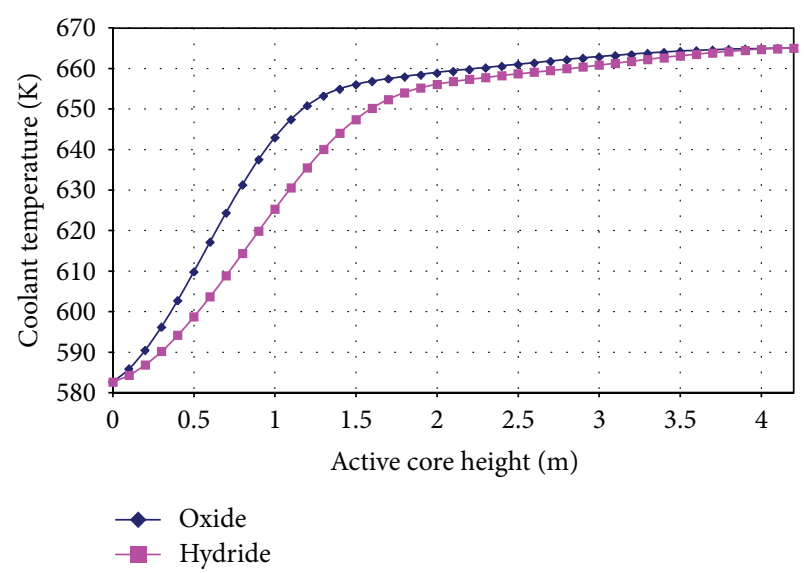

FIGURE 8: Average axial temperature profile in oxide and hydride assemblies.

TABLE 5: Hot channel factors oxide assembly.

\begin{tabular}{llllllll}
\hline 1.014 & 1.010 & 1.004 & 1.000 & 1.000 & 1.004 & 1.010 & 1.014 \\
1.010 & 1.004 & 0.999 & 0.995 & 0.995 & 0.999 & 1.004 & 1.010 \\
1.004 & 0.999 & 1.000 & 0.998 & 0.998 & 1.000 & 0.999 & 1.004 \\
1.000 & 0.995 & 0.997 & & & 0.998 & 0.995 & 1.000 \\
1.000 & 0.995 & 0.997 & & & 0.997 & 0.995 & 1.000 \\
1.004 & 0.998 & 0.999 & 0.997 & 0.997 & 0.999 & 0.998 & 1.004 \\
1.010 & 1.004 & 0.998 & 0.995 & 0.995 & 0.998 & 1.004 & 1.010 \\
1.014 & 1.010 & 1.004 & 1.000 & 1.000 & 1.004 & 1.010 & 1.014 \\
\hline
\end{tabular}

in both assemblies, which has its maximum in the lower core, the cladding temperatures show a peak in this region. In the oxide assembly, the maximum cladding temperature is located at an axial elevation of about $1 \mathrm{~m}$ from core inlet, and the value is about $696 \mathrm{~K}$. In the hydride assembly, the maximum cladding temperature is located at an axial elevation of about $1.5 \mathrm{~m}$ from core inlet. Its value is about $684 \mathrm{~K}$ and 10 degrees lower than in the oxide assembly. Above this position, the coolant specific heat increases sharply when reaching the 


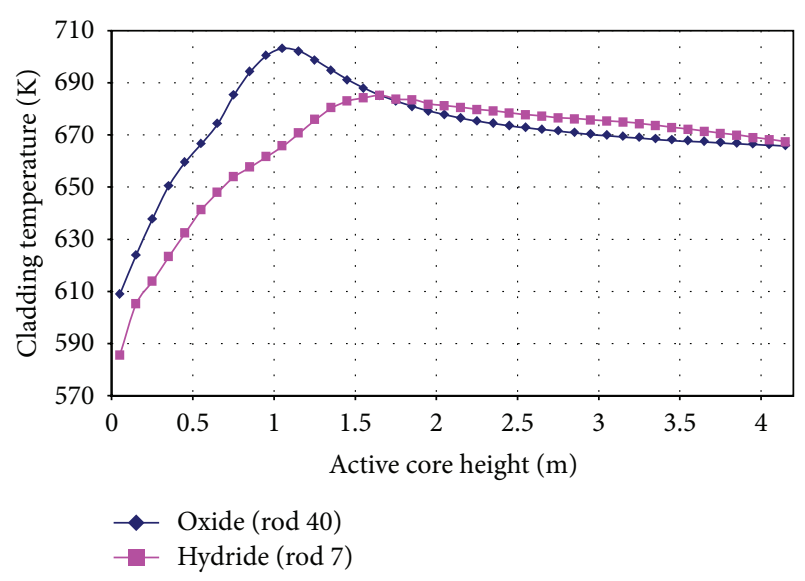

FIgURE 9: Axial cladding temperature profile in oxide and hydride assemblies.

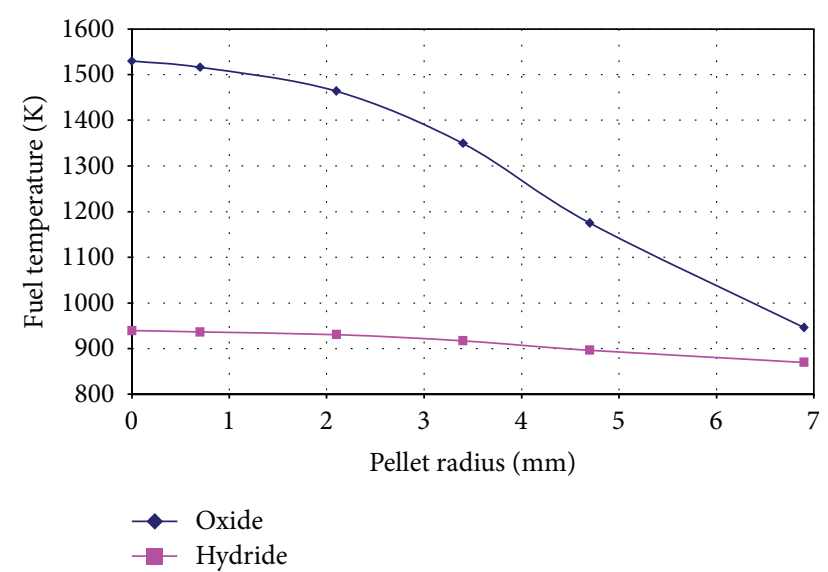

FIGURE 10: Radial pellet temperature profile in oxide and hydride assemblies.

TABLE 6: Hot channel factors hydride assembly.

\begin{tabular}{llllllll}
\hline 1.021 & 1.014 & 1.004 & 0.998 & 0.998 & 1.003 & 1.013 & 1.020 \\
1.014 & 1.001 & 0.993 & 0.989 & 0.988 & 0.993 & 1.001 & 1.013 \\
1.004 & 0.993 & 0.998 & 0.995 & 0.995 & 0.998 & 0.993 & 1.003 \\
0.998 & 0.989 & 0.995 & & & 0.995 & 0.988 & 0.997 \\
0.998 & 0.989 & 0.995 & & & 0.994 & 0.988 & 0.997 \\
1.004 & 0.993 & 0.998 & 0.995 & 0.995 & 0.997 & 0.992 & 1.003 \\
1.014 & 1.001 & 0.993 & 0.988 & 0.988 & 0.993 & 1.000 & 1.012 \\
1.021 & 1.014 & 1.004 & 0.998 & 0.997 & 1.003 & 1.013 & 1.020 \\
\hline
\end{tabular}

pseudocritical point resulting in an enhancement of the heat transfer coefficient. That explains the slight decrease in the cladding temperature shown in this region. Further above this region, the reduction of axial pin power combined with the enhanced heat transfer conditions causes the cladding temperature to remain roughly constant up to the core outlet.

The pellet radial temperature profile at the hottest location in both assemblies is depicted in Figure 10. In the oxide assembly the hottest location is found at the fuel rod position
40 at active height of $950 \mathrm{~mm}$ and in the hydride assembly at the fuel rod position 7 at active height of $1350 \mathrm{~mm}$. The peak pellet temperature in the oxide fuel is $1510 \mathrm{~K}$ whereas in the hydride fuel it is only $938 \mathrm{~K}$. This is mainly due to the higher thermal conductivity of hydride fuel $(22.591 \mathrm{~W} / \mathrm{mK})$ which is a factor of 6.5 of the oxide fuel $(3.4591 \mathrm{~W} / \mathrm{mK})$. The pellet peak temperature in the hydride fuel is below the fuel temperature normal operation limit of $1023 \mathrm{~K}\left(750^{\circ} \mathrm{C}\right)$. There are two potential options for design improvement: an increase in fuel pellet diameter which could help to increase the heavy metal loading and an increase in the linear heat generation rate (LHGR) which could improve the thermal rating.

\section{Summary}

The potential benefit of use of hydride fuel in HPLWR without introducing significant modification in the current core design has been evaluated. Neutronics and thermal hydraulic analyses were performed for a single assembly model of oxide and hydride fuels assuming the current design and operation conditions of the HPLWR concept.

The results of the neutronics analyses show that the HPLWR assembly with hydride fuel exhibits higher moderation with softer neutron spectrum and slightly more uniform axial power distribution.

Both hydride and oxide assembly options achieve a cycle length of 18 months with sufficient excess reactivity. The discharge burnup in the hydride assembly is higher than in the oxide assembly manly due to the higher enrichment. The fuel temperature reactivity coefficient at BOC of the hydride assembly is comparatively improved due the presence of the solid moderator. On the other hand the moderator and void coefficients at BOC are significantly lower.

The thermal hydraulic results show that the fuel temperature in both the oxide and hydride assembly options is well below the design limits. Due the high thermal conductivity of the hydride fuel, the pellet peak temperature remains below the design temperature limit for normal operation of $750^{\circ} \mathrm{C}$.

The current analyses are limited to Beginning of Cycle (BOC) conditions for a fresh assembly and further analyses at End of Cycle (EOC) conditions are indispensable for more comprehensive evaluation.

Under the premise of the current study, to assess the potential benefits of the use of hydride fuel in the current design of the HPLWR, it appears that the achieved improvements in the core neutronics characteristics are not sufficient to compensate the many disadvantages of hydride fuel and to justify the replacement of the oxide fuel. Therefore for a final evaluation of the use of hydride fuels in HPLWR concepts additional studies which include also subassembly and core layout design modification are required. In particular the simplification of the core design and the elimination of the moderate rod in the centre of the fuel bundle could be attractive to HPLWR.

\section{Conflict of Interests}

The authors declare that there is no conflict of interests regarding the publication of this paper. 


\section{References}

[1] D. Squarer, T. Schulenberg, D. Struwe et al., "High performance light water reactor," Nuclear Engineering and Design, vol. 221, no. 1-3, pp. 167-180, 2003.

[2] GA Technologies INC, "Uranium-zirconium hydride TRIGALEU fuel," in Research Reactor Core Conversion Guidebook, Volume 4: Fuels (Appendices I-K), IAEA TECDOC 643/4, 1992.

[3] E. Greenspan, M. Fratoni, F. Ganda et al., "Hydride fuel for LWRs-project overview," Nuclear Engineering and Design, vol. 239, no. 8, pp. 1374-1405, 2009.

[4] Z. Shayer and E. Greenspan, "Preliminary assessment of possibilities for improving the performance of SCWR using hydride fuel," in Proceedings of the 3rd Workshop on Advanced Reactors with Innovative Fuels (ARWIF '05), Oak Ridge, Tenn, USA, February 2005.

[5] J. Hofmeister, C. Waata, J. Starflinger, T. Schulenberg, and E. Laurien, "Fuel assembly design study for a reactor with supercritical water," Nuclear Engineering and Design, vol. 237, no. 14, pp. 1513-1521, 2007.

[6] X-5 Monte Carlo Team, "MCNP_a general Monte Carlo Nparticle transport code, version-5," Tech. Rep. LA-UR-03-1987, Los Alamos National Laboratory, 2003.

[7] J. S. Hendricks, “MCNPX 2.6.0 Manual," Tech. Rep. LA-CP-071473, Los Alamos National Laboratory, 2008.

[8] D. Basile, M. Beghi, R. Chierici, E. Salina, and E. Brega, "COBRA-EN: an upgraded version of the COBRA-3C/MIT code for thermal hydraulic transient analysis of light water reactor fuel assemblies and cores," Tech. Rep. 1010/1, ENELCRTN, Milano, Italy, 1999.

[9] L. Ammirabile, "Studies on supercritical water reactor fuel assemblies using the sub-channel code COBRA-EN," Nuclear Engineering and Design, vol. 240, no. 10, pp. 3087-3094, 2010.

[10] W. Wagner and A. Kruse, "The industrial standard IAPWS-IF97 for the thermodynamic properties and supplementary equations for other properties," in Properties of Water and Steam, Springer, Heidelberg, Germany, 1997.

[11] A. A. Bishop, R. O. Sandberg, and L. S. Tong, "Forced convection heat transfer to water at near critical temperatures and supercritical pressures," WCAP-2056-P, Part-III-B, 1964.

[12] F. W. Dittus and L. M. K. Boelter, Transfer in Automobile Radiators of Tubular Type, vol. 2 (13) of University of California Publications in Engineering, University of California Press, Berkeley, Calif, USA, 1930.

[13] K. Yamagata, K. Nishikawa, S. Hasegawa, T. Fujii, and S. Yoshida, "Forced convective heat transfer to supercritical water flowing in tubes," International Journal of Heat and Mass Transfer, vol. 15, no. 12, pp. 2575-2593, 1972.

[14] N. Aksan, W. Ambrosini, L. Ammirabile et al., "Heat transfer behaviour and thermohydraulics code testing for supercritical water cooled reactors (SCWRs)," IAEA TECDOC 1746, IAEA, Vienna, Austria, 2014.

[15] X. Cheng and T. Schulenberg, "Heat transfer at supercritical pressures-literature review and application to an HPLWR," Scientific Report FZKA 6609, FZK, 2001.

[16] S. Himmel, Modellierung des Strömungsverhaltens in einem HPLWR-Brennelement mit Drahtwendelabstandshaltern [Ph.D. thesis], Universität Stuttgart, 2008.

[17] L. Monti, Multi-scale, coupled reactor physics/thermal-hydraulics system and applications to the HPLWR 3 pass core [Ph.D. thesis], Universität Karlsruhe, 2009.
[18] J. J. Duderstadt and L. J. Hamilton, Nuclear Reactor Analysis, John Wiley \& Sons, 1976. 


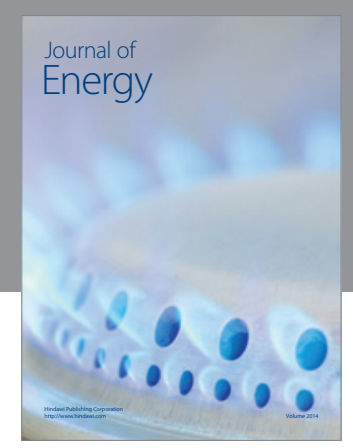

Journal of

Industrial Engineering
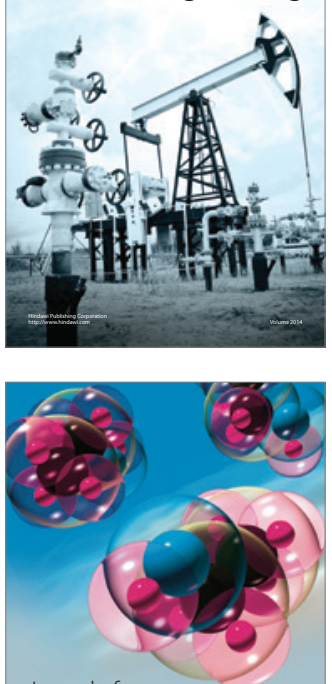

Fuels
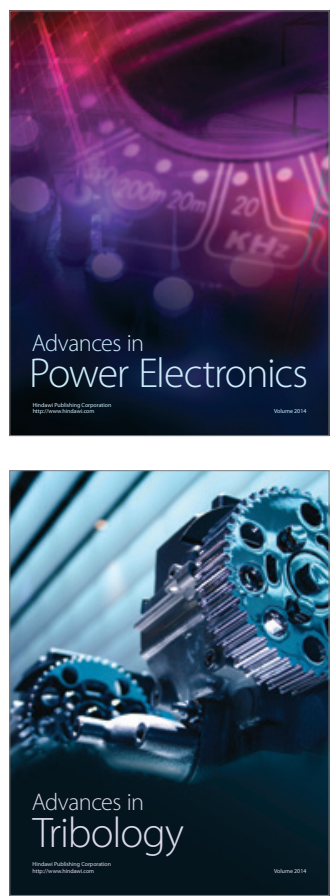

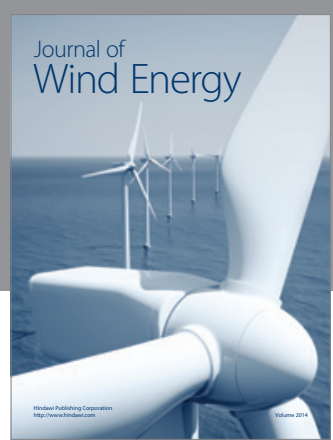

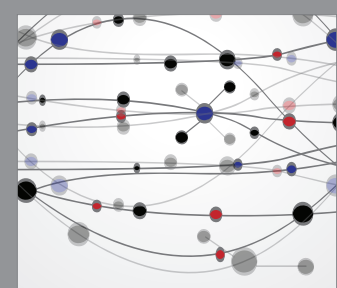

The Scientific World Journal

Submit your manuscripts at http://www.hindawi.com

Journal of

Structures
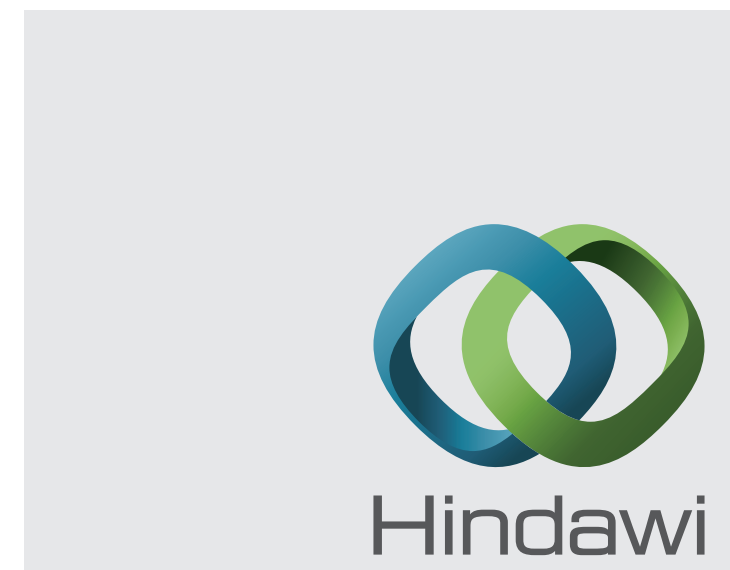

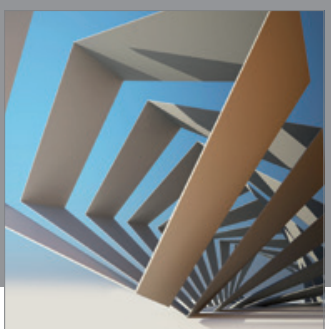

Rotating

Machinery
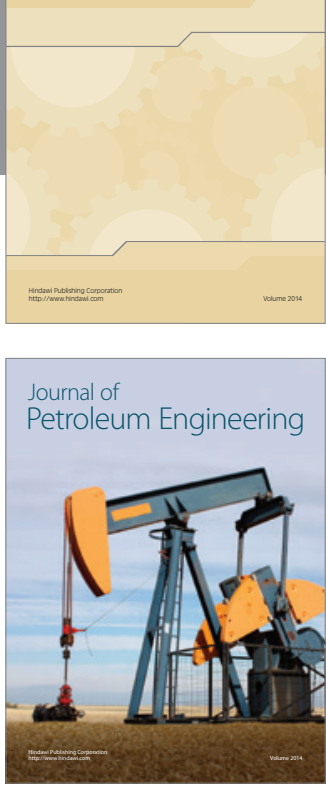

Journal of

Solar Energy
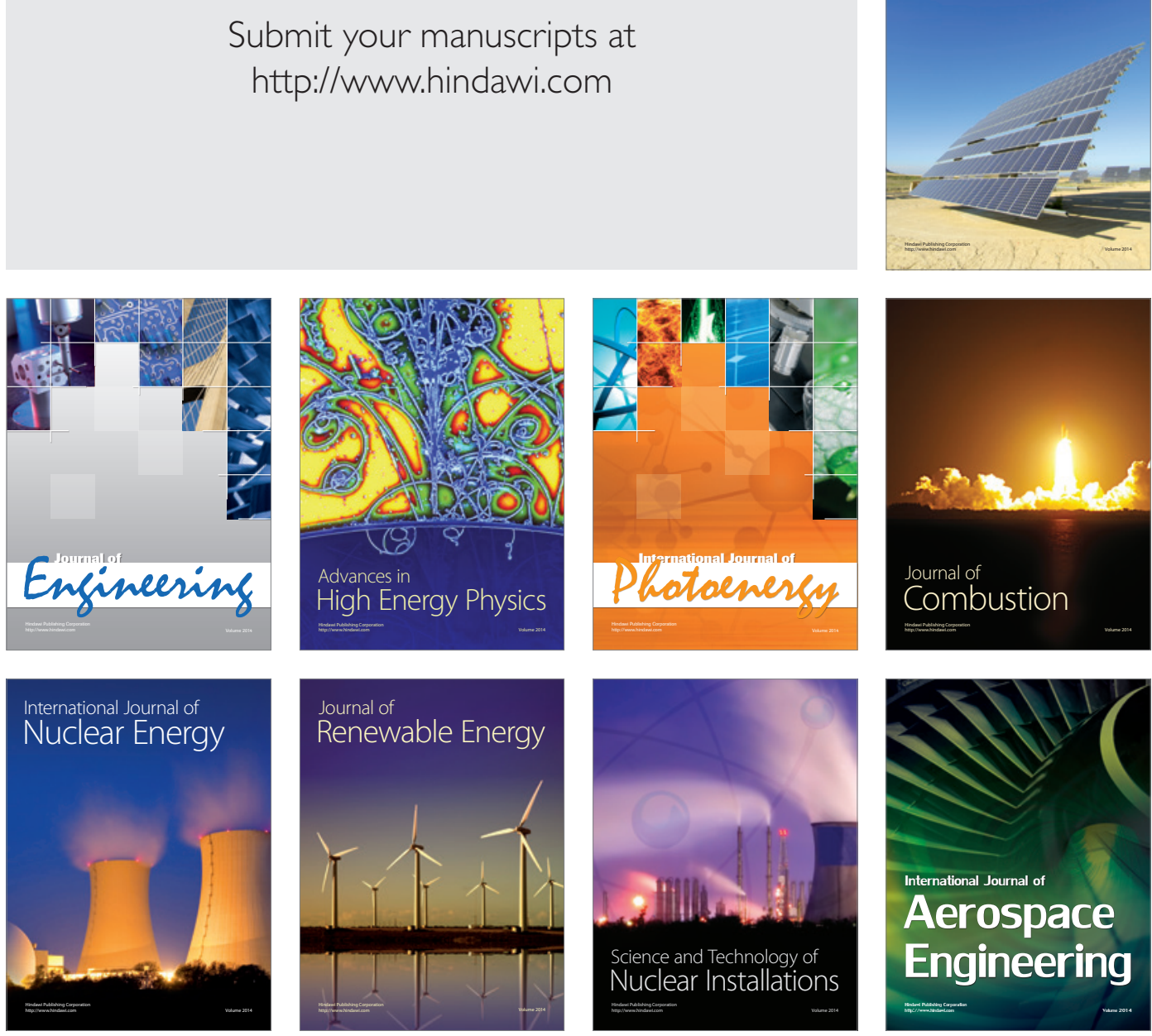